\title{
Relative Attitude Trajectory Tracking Using Line Of Sight Measurements Under Spacecraft Position Dynamics
}

\author{
Rakesh R. Warier* Arpita Sinha* Srikant Sukumar* \\ * Dept. of Systems and Control Engineering, IIT Bombay, India \\ (e-mail: $\{$ rakeshwarier,asinha,srikant\}@sc.iitb.ac.in).
}

\begin{abstract}
:
This paper considers the problem of controlling relative attitude between two spacecrafts, so as to follow a smoothly time varying desired relative attitude trajectory. The spacecrafts are modelled as rigid bodies and attitudes are represented in non-linear state space of $\mathrm{SO}(3)$. The attitude control law proposed does not need relative attitude measurement and is determined from line of sight (LOS) unit vectors measured by spacecrafts to each other and from each spacecraft to a common object in respective body fixed frames. Unlike existing control laws using LOS measurements, positions of spacecrafts are not considered fixed. The state feedback laws proposed in this work guarantee almost global asymptotic tracking of the desired time varying attitude trajectory, when positions of spacecrafts do not become collinear or coincide. The simulation results of the proposed control law are also shown.
\end{abstract}

Keywords: Multi-agent Systems; Distributed Systems; Nonlinear Systems and Control

\section{INTRODUCTION}

Spacecraft formation flying is an enabling technology that is actively pursued by many space agencies. Multiple spacecrafts cooperating together can act as a large virtual instrument which will be more powerful and robust than a monolithic spacecraft. However satellites in formation flying missions are required to work together, and this necessitates that they maintain some specific relative position and attitude configuration relative to each other (Scharf et al. (2004)). We here consider the problem of relative attitude trajectory tracking control, which is vital for achieving formation flying mission objectives.

Most of the existing relative attitude control laws assume absolute attitude of each spacecraft is measured independently with respect to a common inertial frame and are communicated to each other so as to calculate relative attitude. Recently Lee (2012) and Wu et al. (2013) considered the relative attitude control without constructing full attitudes of spacecrafts and comparing them to find relative attitude. In Lee (2012) the control law to achieve desired relative attitude between two spacecrafts is obtained in terms of LOS unit vectors between the spacecrafts and LOS unit vectors from each spacecraft to a common object in the respective body frames. Wu et al. (2013) consider relative attitude trajectory tracking control for multiple spacecrafts. However in Lee (2012) and Wu et al. (2013) it is assumed that the positions of spacecrafts and the common object are fixed and stability proof of the closed loop system depends on this assumption.

In this paper we consider relative attitude trajectory tracking control making use of LOS unit vectors with non fixed position dynamics. As in $\mathrm{Wu}$ et al. (2013), the control law is obtained directly in terms of LOS measurements between two spacecrafts and each spacecraft to a common object. However our proposed control achieves the desired relative attitude tracking control under relative translational dynamics between spacecrafts and the common object, almost asymptotically as long as spacecrafts and the common object do not become collinear or coincide.

\section{PROBLEM FORMULATION}

We consider two spacecrafts and a common object moving in space. Let $r_{1}, r_{2}, r_{3} \in \mathbb{R}^{3}$ be the position vectors of centre of mass of spacecraft 1,2 and the common object respectively, in an inertial frame. The position dynamics of the spacecrafts or the common object is unknown, but it is assumed that spacecrafts and common object never become collinear or coincide. More precisely we assume

(A1) The centre of masses of the three spacecraft are not collinear or coinciding $\left(r_{2}(t)-r_{1}(t)\right) \times\left(r_{3}(t)-r_{2}(t)\right) \neq$ $\left[\begin{array}{lll}0 & 0 & 0\end{array}\right]^{\top}, \forall t>0$.

Assumption $(A 1)$ is necessary because when spacecrafts and common object are collinear, relative attitude between spacecrafts cannot be completely estimated using LOS measurements between them. We describe some of the mathematical preliminaries in the next section and then describe the dynamics of the problem considered.

\subsection{Mathematical Preliminaries}

Spacecraft attitude dynamics are modelled to be rigid body dynamics. State space of a rigid body attitude dynamics is a compact manifold $S O(3)$. However classical approach of attitude control made use of local representations like Euler angles and non unique representations like 
quaternions. It was only recently that rigid body attitude control problems were analysed in $S O(3)$. Some examples of rigid body attitude control in $S O(3)$ is given by Nair and Leonard (2007), Sarlette et al. (2007), Bullo and Murray (1999) and Sanyal and Chaturvedi (2008). The manifold $S O(3)$ is given by

$$
S O(3)=\left\{R \in \mathbb{R}^{3 \times 3} \mid R^{\top} R=R R^{\top}=I, \operatorname{det}(R)=1\right\}
$$

Due to topological properties of $S O(3)$ no globally asymptotically stable equilibrium exists under continuous control (Bhat and Bernstein (2000)). SO(3) forms a Lie group under the group operation matrix multiplication. Lie algebra of $S O(3)$ is denoted as $s o(3)$ and is given by

$$
\text { so }(3)=\left\{S \in \mathbb{R}^{3 \times 3} \mid S=-S^{\top}\right\}
$$

Map $\wedge: \mathbb{R}^{3} \rightarrow s o(3)$ denotes the isomorphism from $\mathbb{R}^{3}$ to so(3). If $x=\left[x_{1}, x_{2}, x_{3}\right]^{T}, x \in \mathbb{R}^{3}$

$$
\hat{x}=\left[\begin{array}{ccc}
0 & -x_{3} & x_{2} \\
x_{3} & 0 & -x_{1} \\
-x_{2} & x_{1} & 0
\end{array}\right]
$$

Further $\hat{x}$ represents the skew symmetric matrix implementing cross product, i.e. $\hat{x} y=x \times y, \forall y$. It's inverse is denoted as $\vee: s o(3) \rightarrow \mathbb{R}^{3}$, implicitly we can define $(\hat{x})^{\vee}=x$. We define a map skew : $\mathbb{R}^{3 \times 3} \rightarrow$ so(3), as skew $(A):=$ $\frac{A-A^{\top}}{2}$. Now $\forall A \in \mathbb{R}^{3 \times 3},(\operatorname{skew}(A))^{\vee}$ is defined. Also let $\operatorname{sym}(A):=\frac{A+A^{\top}}{2}$, notice that $A=\operatorname{sym}(A)+\operatorname{skew}(A)$.

The following results are useful. $\forall a_{1}, a_{2} \in \mathbb{R}^{3}$, and $R \in$ $\mathrm{SO}(3)$,

$$
\begin{aligned}
a_{1} \cdot a_{2} & =\left(R a_{1}\right) \cdot\left(R a_{2}\right) \\
R\left(a_{1} \times a_{2}\right) & =\left(R a_{1}\right) \times\left(R a_{2}\right) \\
R^{\top} \hat{a}_{1} R & =\widehat{R^{\top} a_{1}}
\end{aligned}
$$

$\operatorname{tr}()$ is the trace of a square matrix, defined as sum of its diagonal elements, $\forall A_{1}, A_{2} \in R^{n \times n}$. We refer to Bernstein (2009),

$$
\begin{aligned}
\operatorname{tr}\left(A_{1}^{\top} A_{2}\right) & =\operatorname{tr}\left(A_{2}^{\top} A_{1}\right)=\operatorname{tr}\left(A_{1} A_{2}^{\top}\right) \\
\operatorname{tr}\left(A_{1} A_{2}\right) & =\operatorname{tr}\left(A_{2}^{\top} A_{1}^{\top}\right)=\operatorname{tr}\left(A_{1}^{\top} A_{2}^{\top}\right) \\
\operatorname{tr}\left(A_{1}^{\top} A_{2}\right) & =\sum_{i=1}^{3} \operatorname{col}_{i}\left(A_{1}\right) \cdot \operatorname{col}_{i}\left(A_{2}\right) \\
\operatorname{tr}\left(\hat{a}_{1} \hat{a}_{2}\right) & =2\left(a_{1} \cdot a_{2}\right) \\
\operatorname{tr}\left(\operatorname{sym}\left(A_{1}\right) \hat{a}_{1}\right) & =0
\end{aligned}
$$

here $\operatorname{col}_{i}\left(A_{1}\right)$ is the $i$-th column of matrix $A_{1}$. Also we denote the $3 \times 3$ identity matrix by $I$.

\subsection{Attitude Dynamics}

Equations of motion of the attitudes of $i$-th spacecraft, for $i=1,2$ are given by

$$
\begin{aligned}
\dot{R}_{i} & =R_{i} \hat{\Omega}_{i} \\
J_{i} \dot{\Omega}_{i} & =J_{i} \Omega_{i} \times \Omega_{i}+\tau_{i}
\end{aligned}
$$

where $J_{i} \in \mathbb{R}^{3 \times 3}$ is the moment of inertia, $\Omega_{i} \in \mathbb{R}^{3}$ the angular velocity, and $\tau_{i} \in \mathbb{R}^{3}$ the control torque in of $i$-th spacecraft in its body fixed frame.

We define $Q_{21}$ as the relative attitude of spacecraft 2 with respect to the attitude of spacecraft 1, i.e.

$$
Q_{21}:=R_{1}^{\top} R_{2}
$$

The time derivative of relative attitude $Q_{21}$ is obtained to be

$$
\begin{aligned}
\dot{Q}_{21} & =-R_{1}^{\top} R_{1} \hat{\Omega}_{1} R_{1}^{\top} R_{2}+R_{1}^{\top} R_{2} \hat{\Omega}_{2} \\
& =-\hat{\Omega}_{1} Q_{21}+Q_{21} \hat{\Omega}_{2} \\
& =Q_{21} \hat{\Omega}_{21}
\end{aligned}
$$

where we define $\Omega_{21}$ to be

$$
\Omega_{21}:=\Omega_{2}-Q_{21}^{\top} \Omega_{1}
$$

here we have made use of (6). Let $Q_{21}^{d}(t)$ be a desired relative attitude trajectory of spacecraft 2 with respect to the attitude of spacecraft 1, and is given as a smooth function of time. Then it satisfies kinematic relation,

$$
\dot{Q}_{21}^{d}=Q_{21}^{d} \hat{\Omega}_{21}^{d}
$$

Here $\Omega_{21}^{d}(t)$ is the desired relative angular velocity. We choose $\Omega_{1}^{d}(t)$ and $\Omega_{2}^{d}(t)$ to be smooth trajectories that satisfy,

$$
\Omega_{21}^{d}=\Omega_{2}^{d}-\left(Q_{21}^{d}\right)^{\top} \Omega_{1}^{d}
$$

For example $\Omega_{1}^{d}(t)$ and $\Omega_{2}^{d}(t)$ can be chosen to be

$$
\Omega_{2}^{d}(t)=\frac{1}{2} \Omega_{21}^{d}(t) \quad \Omega_{1}^{d}(t)=-\frac{1}{2} Q_{21}^{d} \Omega_{21}^{d}
$$

From (18), (17) can be written as

$$
\begin{aligned}
\dot{Q}_{21}^{d} & =Q_{21}^{d}\left(\hat{\Omega}_{2}^{d}-\left(Q_{21}^{d}\right)^{\top} \hat{\Omega}_{1}^{d} Q_{21}^{d}\right) \\
& =Q_{21}^{d} \hat{\Omega}_{2}^{d}-\hat{\Omega}_{1}^{d} Q_{21}^{d}
\end{aligned}
$$

Angular velocity errors $e_{\Omega_{i}}, i=1,2$ are defined to be

$$
e_{\Omega_{i}}:=\Omega_{i}-\Omega_{i}^{d}
$$

\subsection{Measured Variables}

We assume that the spacecrafts lack a common frame of reference and do not make any absolute measurements. The spacecrafts 1 and 2 make relative measurements between each other and to the common object, about their body fixed frames, and communicate the same to each other.

Let $(i, j) \in\{(1,2),(2,1),(1,3),(2,3)\}$. We denote the line of sight unit vector observed from the $i$-th spacecraft to the $j$-th spacecraft and represented in the inertial frame as $s_{i j}$. This is given by

$$
s_{i j}=\frac{\left(r_{j}-r_{i}\right)}{\left\|\left(r_{j}-r_{i}\right)\right\|}
$$

Measurements are however made in each spacecraft's own body frame. We define $l_{i j}$ as the line of sight unit vector observed from the $i$-th spacecraft to the $j$-th spacecraft, represented in the $i$-th body fixed frame. This is given by

$$
l_{i j}=R_{i}^{\top} s_{i j}=R_{i}^{\top} \frac{\left(r_{j}-r_{i}\right)}{\left\|\left(r_{j}-r_{i}\right)\right\|}
$$

\subsection{Control Objectives}

We consider two spacecrafts with dynamics given in (12)(13) and reference attitude trajectory given by (17), spacecrafts are able to communicate LOS unit vectors with each other, and position dynamics satisfies (A1). The control objectives are to achieve,

(1) Achieving desired relative attitude between spacecrafts $\lim _{t \rightarrow \infty}\left(R_{1}(t)\right)^{\top} R_{2}(t)=Q_{21}^{d}(t)$, 
(2) Achieving desired angular velocities, $\lim _{t \rightarrow \infty} \Omega_{1}(t)=$ $\Omega_{1}^{d}(t)$ and $\lim _{t \rightarrow \infty} \Omega_{2}(t)=\Omega_{2}^{d}(t)$

using LOS unit vectors $l_{12}, l_{21}, l_{13}$, and $l_{23}$.

\section{RELATIVE ATTITUDE DETERMINATION}

We make use of LOS vectors measured in respective body frames, $l_{12}, l_{21}, l_{13}$ and $l_{23}$ to determine relative attitude. We refer to section 2.2 of chapter 12, Wertz (1978).

$$
\begin{aligned}
& l_{123}:=\frac{\left(l_{12} \times l_{13}\right)}{\left\|l_{12} \times l_{13}\right\|}=\frac{R_{1}^{\top}\left(\left(r_{2}-r_{1}\right) \times\left(r_{3}-r_{1}\right)\right)}{\left\|\left(r_{2}-r_{1}\right) \times\left(r_{3}-r_{2}\right)\right\|} \\
& l_{213}:=\frac{\left(l_{21} \times l_{23}\right)}{\left\|l_{21} \times l_{23}\right\|}=\frac{R_{2}^{\top}\left(\left(r_{1}-r_{2}\right) \times\left(r_{3}-r_{2}\right)\right)}{\left\|\left(r_{1}-r_{2}\right) \times\left(r_{3}-r_{2}\right)\right\|}
\end{aligned}
$$

In addition we have

$$
\begin{aligned}
\left(r_{2}-r_{1}\right) \times\left(r_{3}-r_{1}\right) & =\left(r_{2}-r_{3}+r_{3}-r_{1}\right) \times\left(r_{3}-r_{1}\right) \\
& =\left(r_{3}-r_{1}\right) \times\left(r_{3}-r_{2}\right) \\
\left(r_{1}-r_{2}\right) \times\left(r_{3}-r_{2}\right) & =\left(r_{1}-r_{3}+r_{3}-r_{2}\right) \times\left(r_{3}-r_{2}\right) \\
& =-\left(r_{3}-r_{1}\right) \times\left(r_{3}-r_{2}\right)
\end{aligned}
$$

Since because of assumption (A1), we have $\left(r_{3}-r_{1}\right) \times$ $\left(r_{3}-r_{2}\right) \neq\left[\begin{array}{lll}0 & 0 & 0\end{array}\right]^{\top}$ and $l_{123}$ and $l_{213}$ are well defined. If we define $s_{123}=\frac{\left(r_{3}-r_{1}\right) \times\left(r_{3}-r_{2}\right)}{\left\|\left(r_{3}-r_{1}\right) \times\left(r_{3}-r_{2}\right)\right\|}$, then $l_{123}=R_{1}^{\top}\left(s_{123}\right)$ and $l_{213}=-R_{2}^{\top}\left(s_{123}\right)$, Observe that $s_{123}=s_{12} \times s_{13}$.

Construct a matrix with unit vectors $l_{12}, l_{123}$ and $l_{12} \times l_{123}$ as the column vectors.

$$
\begin{aligned}
P_{1} & :=\left[l_{12}\left(l_{123}\right)\left(l_{12} \times l_{123}\right)\right] \\
& =\left[R_{1}^{\top} s_{12} R_{1}^{\top}\left(s_{123}\right) R_{1}^{\top}\left(s_{12} \times s_{123}\right)\right] \\
& =R_{1}^{\top}\left[s_{12}\left(s_{123}\right)\left(s_{12} \times s_{123}\right)\right]
\end{aligned}
$$

Similarly we can construct a matrix with unit vectors $l_{21}$, $l_{213}$ and $\left(l_{21} \times l_{213}\right)$ as the column vectors

$$
\begin{aligned}
P_{2} & :=\left[l_{21}\left(l_{213}\right)\left(l_{213}\right) \times l_{21}\right] \\
& =-\left[R_{2}^{\top} s_{12} R_{2}^{\top}\left(s_{123}\right) R_{2}^{\top}\left(s_{12} \times s_{123}\right)\right] \\
& =-R_{2}^{\top}\left[s_{12}\left(s_{123}\right)\left(s_{12} \times s_{123}\right)\right]
\end{aligned}
$$

Note that columns vectors of both $P_{1}$ and $P_{2}$ form orthogonal bases, and thus $P_{1}$ and $P_{2}$ are orthogonal matrices. Hence

$$
P_{1}^{-1}=P_{1}^{\top} \quad P_{2}^{-1}=P_{2}^{\top}
$$

From (27) and (28),

$$
\left[s_{12}\left(s_{123}\right)\left(s_{12} \times s_{123}\right)\right]=-R_{2} P_{2}=R_{1} P_{1}
$$

We can determine the relative attitude $Q_{12}:=R_{1}^{\top} R_{2}$ using (28), to be

$$
Q_{21}=R_{1}^{\top} R_{2}=-P_{1} P_{2}^{\top}
$$

The attitude determination scheme is not explicitly used in the control law. We make use of the scheme to show that our control law indeed achieves tracking of desired relative attitude trajectory.

\section{ERROR FUNCTIONS}

We make use of error functions to design the control law. Error functions are chosen such that minimizing of error functions will achieve the control objectives.

\subsection{Attitude Error Function}

Trace and modified trace functions are very commonly used in attitude control design. Some examples from the literature include Sanyal and Chaturvedi (2008), Sarlette (2009) and Nair and Leonard (2007). For $Q_{1}, Q_{2} \in S O(3)$, $\operatorname{tr}\left(Q_{1}^{\top} Q_{2}\right)=1+2 \cos (\theta)$, where $\theta$ is the angle of single axis rotation between $Q_{1}$ and $Q_{2} \cdot \operatorname{tr}\left(Q_{1}^{\top} Q_{2}\right)$ obtains maximum value when $Q_{1}=Q_{2}$. It is known that Let

$$
\begin{aligned}
\Psi_{1}\left(R_{1}, R_{2}\right) & =\operatorname{tr}\left(I-\left(Q_{21}^{d}\right)^{\top} R_{1}^{\top} R_{2}\right) \\
& =\operatorname{tr}\left(I-\left(Q_{21}^{d}\right)^{\top} Q_{21}\right)=\operatorname{tr}(I-X)
\end{aligned}
$$

where

$$
X:=\left(Q_{21}^{d}\right)^{\top} Q_{21}
$$

It can be seen that $\Psi_{1} \geq 0$ and equal to zero only when $Q_{21}^{d}=Q_{21}$. Taking derivatives we have (from lemma A.1, Bullo and Murray (1999))

$$
\dot{\Psi_{1}}=-\operatorname{tr}(\dot{X})
$$

we also have

$$
\begin{aligned}
\dot{X}= & -\left(Q_{21}^{d}\right)^{\top} Q_{21}^{d} \hat{\Omega}_{21}^{d}\left(Q_{21}^{d}\right)^{\top} Q_{21} \\
& +\left(Q_{21}^{d}\right)^{\top} Q_{21} \hat{\Omega}_{21} \\
= & \left(Q_{21}^{d}\right)^{\top} Q_{21} \hat{\Omega}_{21}-\hat{\Omega}_{21}^{d}\left(Q_{21}^{d}\right)^{\top} Q_{21} \\
= & X \hat{\Omega}_{21}-\hat{\Omega}_{21}^{d} X
\end{aligned}
$$

Now

$$
\begin{aligned}
\dot{\Psi}_{1}= & -\operatorname{tr}\left(X \hat{\Omega}_{21}\right)+\operatorname{tr}\left(\hat{\Omega}_{21}^{d} X\right) \\
= & -\operatorname{tr}\left((\operatorname{skew}(X)+\operatorname{sym}(X)) \hat{\Omega}_{21}\right) \\
& +\operatorname{tr}\left(\hat{\Omega}_{21}^{d}(\operatorname{sym}(X)+\operatorname{skew}(X))\right)
\end{aligned}
$$

By (11) we have

$$
\begin{aligned}
\dot{\Psi}_{1}= & -\operatorname{tr}\left((\operatorname{skew}(X)) \hat{\Omega}_{21}\right) \\
& +\operatorname{tr}\left(\hat{\Omega}_{21}^{d}(\operatorname{skew}(X))\right) \\
& -\operatorname{tr}\left(\hat{\Omega}_{2} X\right)-\operatorname{tr}\left(Q_{21}^{\top} \hat{\Omega}_{1} Q_{21}^{\top} X\right) \\
& -\operatorname{tr}\left(\hat{\Omega}_{2}^{d} X\right)+\operatorname{tr}\left(\left(Q_{21}^{d}\right)^{\top} \hat{\Omega}_{2}^{d}\left(Q_{21}^{d}\right)^{\top} X\right) \\
= & 2 e_{\Omega_{1}} \cdot \operatorname{skew}\left(Q_{21}^{d} Q_{21}^{\top}\right)+e_{\Omega_{2}} \cdot \operatorname{skew}\left(\left(Q_{21}^{d}\right)^{\top} Q_{21}\right)
\end{aligned}
$$

The last expression is obtained using (10) and (8). The critical points of function $\operatorname{tr}(I-X)$ are given by (from Sanyal and Chaturvedi (2008))

$$
X \in\{I, \operatorname{diag}[1,-1,-1], \quad \operatorname{diag}[-1,-1,1],
$$

Also at $X=I$, the $\Psi_{1}$ function is at its minimum and at other critical points it achieves the maximum value of 4 .

The following lemma helps us to find derivative of $\Psi_{1}$ in terms of LOS vectors

Lemma 1. Let $A, B \in \mathbb{R}^{3 \times 3}$, Then

$$
\begin{aligned}
\left(2 \operatorname{skew}\left(A B^{\top}\right)\right)^{\vee} & =\left(A B^{\top}-B A^{\top}\right)^{\vee} \\
& =-(a \times x)-(b \times y)-(c \times z)
\end{aligned}
$$

where $a, b, c$ are column vectors of $A$ and $x, y, z$ column vectors of $B$.

Proof. Let $\Gamma=\left(A B^{\top}-B A^{\top}\right)$. Clearly $\Gamma$ is skew symmetric, thus has diagonal elements zero. Non diagonal elements of $\Gamma$ are obtained to be 


$$
\begin{aligned}
(\Gamma)_{12}= & \left(a_{1} x_{2}-a_{2} x_{1}\right)+\left(b_{1} y_{2}-b_{2} y_{1}\right) \\
& +\left(c_{1} z_{2}-c_{2} z_{1}\right) \\
(\Gamma)_{13}= & \left(a_{1} x_{3}-a_{3} x_{1}\right)+\left(b_{1} y_{3}-b_{3} y_{1}\right) \\
& +\left(c_{1} z_{3}-c_{3} z_{1}\right) \\
(\Gamma)_{23}= & \left(a_{2} x_{3}-a_{3} x_{2}\right)+\left(b_{2} y_{3}-b_{3} y_{2}\right) \\
& +\left(c_{2} z_{3}-c_{3} z_{2}\right)
\end{aligned}
$$

And by definition of $\vee$ map we have

$$
(\Gamma)^{\vee}=\left[\begin{array}{r}
-(\Gamma)_{23} \\
(\Gamma)_{13} \\
-(\Gamma)_{12}
\end{array}\right]
$$

Now notice the standard expression of vector product given by,

$$
\left[\begin{array}{l}
a_{1} \\
a_{2} \\
a_{3}
\end{array}\right] \times\left[\begin{array}{l}
x_{1} \\
x_{2} \\
x_{3}
\end{array}\right]=\left[\begin{array}{c}
a_{2} x_{3}-a_{3} x_{2} \\
-\left(a_{1} x_{3}-a_{3} x_{1}\right) \\
a_{1} x_{2}-a_{2} x_{1}
\end{array}\right]
$$

Substituting (40)-(42) in (43) and comparing terms with (44), the identity (39) is evident.

$$
\begin{aligned}
\operatorname{skew}\left(\left(Q_{21}^{d}\right)^{\top} Q_{21}\right) & =2 \operatorname{skew}\left(\left(Q_{21}^{d}\right)^{\top} R_{1}^{\top} R_{2}\right) \\
& =-2 \operatorname{skew}\left(\left(Q_{21}^{d}\right)^{\top} P_{1} P_{2}^{\top}\right) \\
& =-2 \operatorname{skew}\left(\left(\left(Q_{21}^{d}\right)^{\top} P_{1}\right) P_{2}^{\top}\right)
\end{aligned}
$$

Now making use of lemma 1 with $A=\left(Q_{21}^{d}\right)^{\top} P_{1}$ and $B=P_{2}$ we have

$$
\begin{aligned}
2 \operatorname{skew}\left(\left(Q_{21}^{d}\right)^{\top} Q_{21}\right)= & \left(\left(Q_{21}^{d}\right)^{\top} l_{12}\right) \times l_{21} \\
& +\left(\left(Q_{21}^{d}\right)^{\top} l_{123}\right) \times l_{213} \\
& -\left(\left(Q_{21}^{d}\right)^{\top}\left(l_{12} \times l_{123}\right)\right) \times\left(l_{21} \times l_{213}\right)
\end{aligned}
$$

Remember $\operatorname{col}_{i}\left(A_{1} A_{2}\right)=A_{1} \operatorname{col}_{i}\left(A_{2}\right), A_{1}, A_{2}$ are square matrices and $\operatorname{col}_{i}\left(A_{1}\right)$ indicate $i$-th column of matrix $A_{1}$. Now

$$
\begin{aligned}
2 \operatorname{skew}\left(\left(Q_{21}^{d}\right) Q_{21}^{\top}\right) & =2 \operatorname{skew}\left(\left(Q_{21}^{d}\right) R_{2}^{\top} R_{1}\right) \\
& =-2 \operatorname{skew}\left(\left(Q_{21}^{d}\right)^{\top} P_{2} P_{1}^{\top}\right) \\
& =-2 \operatorname{skew}\left(\left(\left(Q_{21}^{d}\right) P_{2}\right) P_{1}^{\top}\right)
\end{aligned}
$$

Making use of lemma 1 with $A=\left(Q_{21}^{d}\right) P_{2}$ and $B=P_{1}$ we have

$$
\begin{aligned}
2 \operatorname{skew}\left(\left(Q_{21}^{d}\right) Q_{21}^{\top}\right)= & \left(\left(Q_{21}^{d}\right) l_{21}\right) \times l_{12} \\
& +\left(\left(Q_{21}^{d}\right) l_{213}\right) \times l_{123} \\
& -\left(\left(Q_{21}^{d}\right)\left(l_{21} \times l_{213}\right)\right) \times\left(l_{12} \times l_{123}\right)
\end{aligned}
$$

Finally we obtain

$$
\begin{aligned}
\dot{\Psi}_{1} & =e_{\Omega_{1}} \cdot\left(\left(Q_{21}^{d}\right)^{\top} l_{12}\right) \times l_{21} \\
& +e_{\Omega_{1}} \cdot\left(\left(Q_{21}^{d}\right)^{\top} l_{123}\right) \times l_{213} \\
& -e_{\Omega_{1}} \cdot\left(\left(Q_{21}^{d}\right)^{\top}\left(l_{12} \times l_{123}\right)\right) \times\left(l_{21} \times l_{213}\right) \\
& +e_{\Omega_{2}} \cdot\left(\left(Q_{21}^{d} l_{21}\right) \times l_{12}\right) \\
& +e_{\Omega_{2}} \cdot\left(\left(Q_{21}^{d} l_{213}\right) \times l_{123}\right) \\
& -e_{\Omega_{2}} \cdot\left(\left(Q_{21}^{d}\left(l_{21} \times l_{213}\right)\right) \times\left(l_{12} \times l_{123}\right)\right)
\end{aligned}
$$

It is interesting to see $\Psi_{1}$ in terms of the attitude determination scheme (31)

$$
\begin{aligned}
\Psi_{1}= & \operatorname{tr}\left(I-\left(Q_{21}^{d}\right)^{\top} R_{1}^{\top} R_{2}\right)=\operatorname{tr}(I)+\operatorname{tr}\left(\left(Q_{21}^{d}\right)^{\top} P_{1} P_{2}^{\top}\right) \\
= & 3+\operatorname{tr}\left(\left(\left(Q_{21}^{d}\right)^{\top} P_{1}\right)^{\top} P_{2}\right) \\
= & \left(1+\left(\left(Q_{21}^{d}\right)^{\top} l_{12}\right) \cdot l_{21}\right)+\left(1+\left(\left(Q_{21}^{d}\right)^{\top} l_{123}\right) \cdot l_{213}\right) \\
& +\left(1-\left(\left(Q_{21}^{d}\right)^{\top}\left(l_{12} \times l_{123}\right)\right) \cdot\left(l_{21} \times l_{213}\right)\right)
\end{aligned}
$$

Expression of $\Psi_{1}$ is obtained in terms of LOS unit vectors. This is desirable for controlling attitude using LOS unit vectors. In Warier and Sinha (2013), the attitude alignment error function is $\left(1+l_{12} \cdot l_{21}\right)$. In Lee (2012), the error functions used are of linear combination of $(1+$ $\left.\left(\left(Q_{21}^{d}\right)^{\top} l_{12}\right) \cdot l_{21}\right)$ and $\left(1+\left(\left(Q_{21}^{d}\right)^{\top} l_{123}\right) \cdot l_{213}\right)$. It can be seen that our expression has additional term compared to Lee (2012).

\subsection{Angular Velocity Error Function}

We chose angular velocity error function of the form

$$
\begin{aligned}
\Psi_{2}\left(\Omega_{1}, \Omega_{2}, \Omega_{1}^{d}, \Omega_{2}^{d}\right) & =\sum_{i=1}^{2}\left(\Omega_{i}-\Omega_{i}^{d}\right)^{\top} J_{i}\left(\Omega_{i}-\Omega_{i}^{d}\right) \\
& =\sum_{i=1}^{2}\left(e_{\Omega_{i}}\right)^{\top} J_{i} e_{\Omega_{i}}
\end{aligned}
$$

Clearly $\Psi_{2}$ is positive definite about error variables $\left(\Omega_{1}-\right.$ $\left.\Omega_{1}^{d}\right)$ and $\left(\Omega_{2}-\Omega_{2}^{d}\right)$. Now

$$
\begin{aligned}
\frac{d}{d t}\left(e_{\Omega_{i}}^{\top} J_{i} e_{\Omega_{i}}\right)= & \left(e_{\Omega_{i}}\right)^{\top}\left(\left(J_{i} \Omega_{i}\right) \times \Omega_{i}+\tau_{i}-e_{\Omega_{i}}^{\top} J_{i} \dot{\Omega}_{i}^{d}\right) \\
= & e_{\Omega_{i}}^{\top}\left(\left(J_{i}\left(e_{\Omega_{i}}+\Omega_{i}^{d}\right)\right) \times\left(e_{\Omega_{i}}+\Omega_{i}^{d}\right)\right) \\
& +e_{\Omega_{i}}^{\top}\left(\tau_{i}-J_{i} \dot{\Omega}_{i}^{d}\right) \\
= & e_{\Omega_{i}}^{\top}\left(J_{i}\left(e_{\Omega_{i}}+\Omega_{i}^{d}\right) \times\left(\Omega_{i}^{d}\right)+\tau_{i}-J_{i} \dot{\Omega}_{i}^{d}\right)
\end{aligned}
$$

Thus we have

$$
\dot{\Psi}_{2}=\sum_{i=1}^{2} e_{\Omega_{i}}^{\top}\left(J_{i}\left(e_{\Omega_{i}}+\Omega_{i}^{d}\right) \times\left(\Omega_{i}^{d}\right)+\tau_{i}-J_{i} \dot{\Omega}_{i}^{d}\right)
$$

Also by definition of $e_{\Omega_{i}}$ we have $e_{\Omega_{i}}+\Omega_{i}^{d}=\Omega_{i}$ Thus

$$
\dot{\Psi}_{2}=\sum_{i=1}^{2} e_{\Omega_{i}}^{\top}\left(J_{i}\left(\Omega_{i}\right) \times\left(\Omega_{i}^{d}\right)+\tau_{i}-J_{i} \dot{\Omega}_{i}^{d}\right)
$$

\section{RELATIVE ATTITUDE TRACKING CONTROL}

In this section we describe our control law and prove the stability results.

\subsection{Control Law}

$$
\begin{aligned}
\tau_{1}= & -k_{\Omega_{1}} e_{\Omega_{1}}-k_{1}\left(Q_{21}^{d} l_{21}\right) \times l_{12}-k_{1}\left(Q_{21}^{d} l_{213}\right) \times l_{123} \\
& +k_{1}\left(Q_{21}^{d}\left(l_{21} \times l_{213}\right)\right) \times\left(l_{12} \times l_{123}\right) \\
& -\left(J_{1}\left(\Omega_{1}\right)\right) \times \Omega_{1}^{d}+J_{1} \dot{\Omega}_{1}^{d} \\
\tau_{2}= & -k_{\Omega_{2}} e_{\Omega_{2}}-k_{1}\left(\left(Q_{12}^{d}\right)^{\top} l_{12}\right) \times l_{21} \\
& -k_{1}\left(\left(Q_{12}^{d}\right)^{\top} l_{123}\right) \times l_{213} \\
& +k_{1}\left(\left(Q_{12}^{d}\right)^{\top}\left(l_{12} \times l_{123}\right)\right) \times\left(l_{21} \times l_{213}\right) \\
& -\left(J_{2}\left(\Omega_{2}\right)\right) \times \Omega_{2}^{d}+J_{2} \dot{\Omega}_{2}^{d}
\end{aligned}
$$

where the control gains

$$
k_{\Omega_{1}}, k_{\Omega_{2}}, k_{1}>0
$$




\subsection{Stability Results}

Theorem 2. Consider the system with states $\left(X, e_{\Omega_{1}}, e_{\Omega_{2}}\right)$ defined as in (34) and (22) with dynamics given by (12)-(13) and (17). Let position dynamics satisfy the assumption (A1). Now under the control law given by (52)(53), following properties hold:

(i) The equilibrium configurations of system are given by

$$
\begin{aligned}
& \mathcal{M}_{1}=\{(I, 0,0)\} \\
& \mathcal{M}_{2}=\{(X, 0,0), X \in \mathcal{N}\}
\end{aligned}
$$

where

$\mathcal{N}=\{\operatorname{diag}[1,-1,-1], \operatorname{diag}[-1,-1,1], \operatorname{diag}[-1,1,-1]\}$

(ii) The desired equilibrium configuration $\mathcal{M}_{1}$ is asymptotically stable and a conservative region of attraction of $\mathcal{M}_{1}$ is given by

$$
\begin{array}{r}
\Psi_{1}(0)<4 k_{1} \\
\sum_{i=1}^{2} \lambda_{\max }\left(J_{i}\right)\left\|e_{\Omega_{i}}\right\|^{2}<4 k_{1}-\Psi_{1}(0)
\end{array}
$$

where $\lambda_{\max }$ represents the largest eigen value.

(iii) Undesired equilibrium configuration $\mathcal{M}_{2}$ is unstable.

Proof. We make use of the La Salle's invariance principle and Chetaev's instability theorem for the proof.

(i) Consider the Lyapunov function

$$
\mathcal{V}=k_{1} \Psi_{1}+\Psi_{2}=k_{1}(\operatorname{tr}(I-X))+\sum_{i=1}^{2} e_{\Omega_{i}}^{\top}\left(J_{i} e_{\Omega_{i}}\right)
$$

Clearly $\mathcal{V} \geq 0$, and $\mathcal{V}=0$ only when the desired control objectives are satisfied. $\mathcal{V}$ is positive definite with respect to variables $I-X, e_{\Omega_{1}}$ and $e_{\Omega_{2}}$.

Now taking the derivative we have,

$$
\dot{\mathcal{V}}=k_{1} \dot{\Psi}_{1}+\dot{\Psi}_{2}
$$

Substituting derivatives of error functions,

$$
\begin{aligned}
\dot{\mathcal{V}} & =k_{1} e_{\Omega_{1}} \cdot\left(\left(Q_{21}^{d}\right)^{\top} l_{12}\right) \times l_{21} \\
& +k_{1} e_{\Omega_{1}} \cdot\left(\left(Q_{21}^{d}\right)^{\top} l_{123}\right) \times l_{213} \\
& -k_{1} e_{\Omega_{1}} \cdot\left(\left(\left(Q_{21}^{d}\right)^{\top}\left(l_{12} \times l_{123}\right)\right) \times\left(l_{21} \times l_{213}\right)\right) \\
& +k_{1} e_{\Omega_{2}} \cdot\left(\left(Q_{21}^{d} l_{21}\right) \times l_{12}\right) \\
& +k_{1} e_{\Omega_{2}} \cdot\left(\left(Q_{21}^{d} l_{213}\right) \times l_{123}\right) \\
& -k_{1} e_{\Omega_{2}} \cdot\left(\left(Q_{21}^{d}\left(l_{21} \times l_{213}\right)\right) \times\left(l_{12} \times l_{123}\right)\right) \\
& +\sum_{i=1}^{2} e_{\Omega_{i}}^{\top}\left(J_{i}\left(e_{\Omega_{i}}+\Omega_{i}^{d}\right) \times\left(\Omega_{i}^{d}\right)+\tau_{i}-J_{i} \dot{\Omega}_{i}^{d}\right)
\end{aligned}
$$

Substituting control terms from (52)-(53) we obtain,

$$
\dot{\mathcal{V}}=-k_{\Omega_{1}}\left\|e_{\Omega_{1}}\right\|^{2}-k_{\Omega_{2}}\left\|e_{\Omega_{2}}\right\|^{2}
$$

Thus we have

$$
\dot{\mathcal{V}} \leq 0
$$

$\mathcal{V}$ is bounded from below and $\dot{\mathcal{V}} \leq 0$, which implies that $\lim _{t \rightarrow \infty} \mathcal{V}(t)$ exists by monotonicity. To apply La Salle's invariance principle, we construct

$$
\begin{aligned}
\mathcal{K}= & \left\{\left(X, e_{\Omega_{1}}, e_{\Omega_{2}}\right), \in S O(3) \times \mathbb{R}^{6} \mid\right. \\
& \left.\mathcal{V}\left(X(t), e_{\Omega_{1}}(t), e_{\Omega_{2}}(t)\right) \leq \mathcal{V}\left(X(0), e_{\Omega_{1}}(0), e_{\Omega_{2}}\right)(0)\right\}
\end{aligned}
$$

$\mathcal{K}$ forms an invariant set in $S O(3) \times \mathbb{R}^{6}$. Now by La Salle's invariance principle, system dynamics converge asymptotically to the largest positively invariant set in subset of $\mathcal{K}$ where $\dot{\mathcal{V}}=0$.

$$
\dot{\mathcal{V}}^{-1}(0)=\{(X, 0,0) \in \mathcal{K} \mid X \in \mathcal{N} \cup I\}
$$

Largest invariant set in $\dot{\mathcal{V}}^{-1}(0)$, are obtained to be

$$
\overline{\mathcal{M}}=\left\{\left(X, e_{\Omega_{1}}, e_{\Omega_{2}}\right) \mid \quad X \in I \cup \mathcal{N}, e_{\Omega_{1}}=e_{\Omega_{2}}=0\right\}
$$

(ii) $\mathcal{V}$ is positive definite and zero only at $\mathcal{M}_{1}$ and $\dot{\mathcal{V}} \leq 0$, which gives us Lyapunov stability of desired equilibrium. Now for all the undesired equilibrium configurations, we have $\Psi_{1}=4$. Under (54) and (55),

$$
\mathcal{V}(0) \leq 4 k_{1}
$$

Since $\dot{\mathcal{V}} \leq 0$, we have

$$
0 \leq \mathcal{V}(t)<\mathcal{V}(0)<4 k_{1}
$$

This guarantees that when initial conditions satisfy (54) and (55) the undesired equilibrium configuration is avoided and system dynamics converge to desired configuration $\mathcal{M}_{1}$.

(iii) Define

$$
\mathcal{W}=4 k_{1}-\mathcal{V}
$$

At the undesired configuration $\mathcal{M}_{2}$, we have $\mathcal{W}=0$. Now we can choose an arbitrarily close region to $\mathcal{M}_{2}$ where $e_{\Omega_{1}}$ or $e_{\Omega_{2}} \neq 0$ and the function $\mathcal{W}>0$. Now $\dot{\mathcal{W}}=-\dot{\mathcal{V}}>0$ (strictly greater than zero because we chose $e_{\Omega_{1}}$ or $\left.e_{\Omega_{2}} \neq 0\right)$. Thus there exists at any arbitrarily small neighbourhood of the undesired equilibrium, a solution trajectory that will escape, which gives that undesired equilibrium is unstable (Khalil (2002), Theorem 3.3).

Also by following similar arguments as in Bayadi and Banavar (2013) it can be shown that the union of region of attractions of unstable equilibria are of lower dimension than $S O(3) \times \mathbb{R}^{6}$.

\section{SIMULATION RESULTS}

For numerical simulation we assumed the position dynamics of the two spacecrafts and common object to be of following form,

$$
\begin{aligned}
& \dot{r}_{i}=v_{i} \\
& \dot{v}_{i}=k_{a}\left(2 \omega \times r_{i}+\omega \times v_{i}\right)
\end{aligned}
$$

where $\omega=\left[\begin{array}{lll}0 & 0 & 0.9\end{array}\right]^{\top}, k_{a}=0.01$, and $i=1,2,3$. The position trajectories follow spiralling out motion. Let $a=\frac{1}{\sqrt{14}}[1,2,3]^{\top}$, and $\grave{a}=\frac{1}{\sqrt{3}}[1,1,-1]^{\top}$. The desired relative attitude dynamics are chosen to be,

$$
Q_{21}^{d}(0)=\exp (\pi \hat{a}) ; \quad \Omega_{21}^{d}(t)=[1-11]^{\prime}
$$

where exp is the matrix exponential.

Numerical simulations are carried out with following initial conditions. Moments of inertia of the two spacecraft are taken to be $J_{1}=J_{2}=\operatorname{diag}[2,3,5] \mathrm{Nm}^{2} . r_{1}(0)=$ $5[\cos (0) \sin (0) \quad 0]^{\top} \mathrm{m}, r_{2}(0)=5\left[\begin{array}{ccc}\cos (120) & \sin (120) & 0\end{array}\right]^{\top} \mathrm{m}$, $r_{3}(0)=5[\cos (240) \sin (240) 0]^{\top} \mathrm{m}, v_{1}(0)=0.1(\omega+\omega \times$ $\left.r_{1}\right) \mathrm{m} / \mathrm{s}, v_{2}(0)=0.1\left(\omega+\omega \times r_{2}\right) \mathrm{m} / \mathrm{s}$, and $v_{3}(0)=0.1(\omega+$ 
$\left.\omega \times r_{3}\right) \mathrm{m} / \mathrm{s}$. The position dynamics viewed in two different angles is shown in figures 1 .

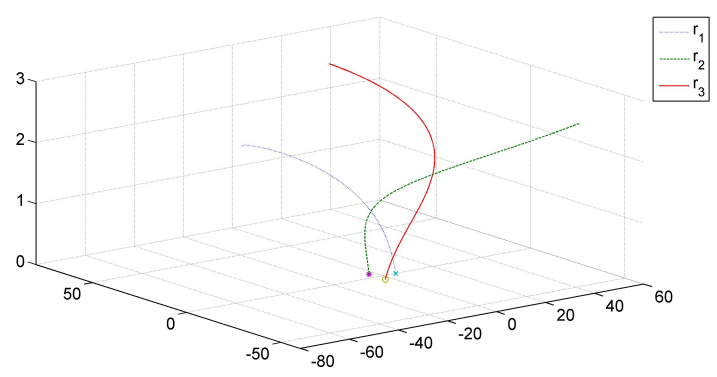

(a)

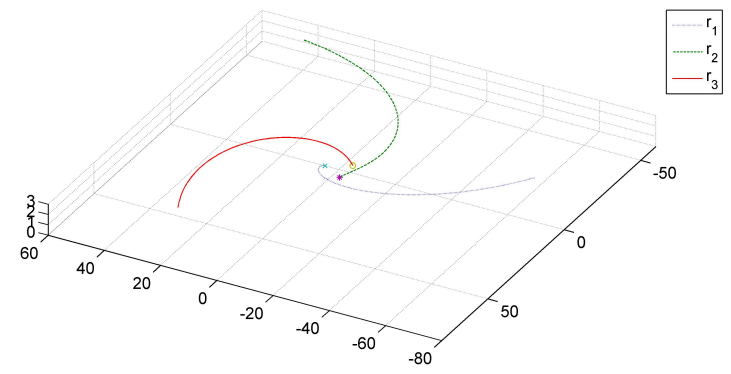

(b)

Fig. 1. Position dynamics of the spacecrafts and common object with respect to time

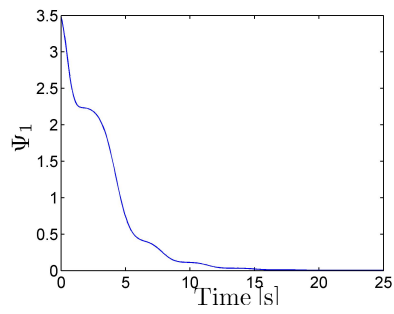

(a) Variation of attitude tracking error function $\Psi_{1}$, with time

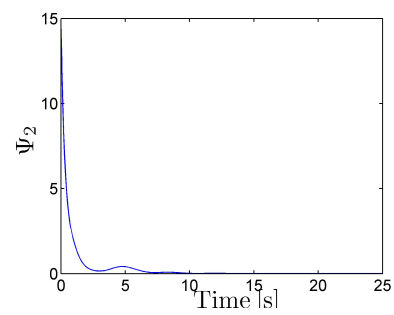

(b) Variation of angular velocity tracking error function $\Psi_{2}$, with time

Fig. 2. Error functions with respect to time

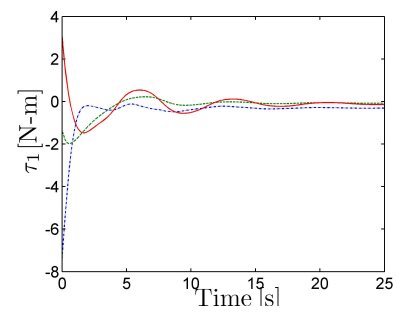

(a) Control torque applied to spacecraft 1

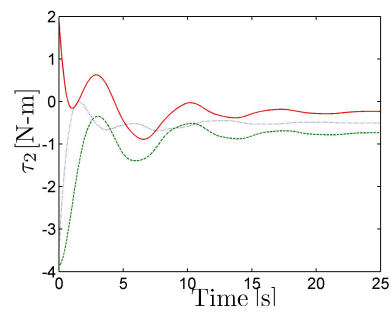

(b) Control torque applied to $\mathrm{s}$ pacecraft 2
Fig. 3. The torque inputs applied to the two spacecraft. (First component: dotted, second component: dashed, third component: solid)

Initial orientation of spacecraft 1 was chosen to be $R_{1}(0)=\exp \left(\frac{4 \pi}{3} \hat{a}\right)$, with initial angular velocity $\Omega_{1}(0)=$ $[2,-0.1,0.5]^{\top} \mathrm{rad} / \mathrm{s}$. Initial conditions for spacecraft 2 , $\Omega_{2}(0)=[1,0.7,0.3]^{\top} \mathrm{rad} / \mathrm{s}$ and $R_{2}(0)=\exp (0.99 \pi \hat{\hat{a}})$.

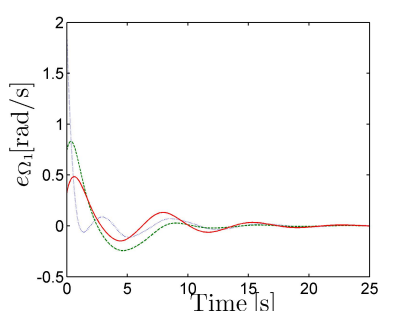

(a) Angular velocity tracking error $e_{\Omega_{1}}$

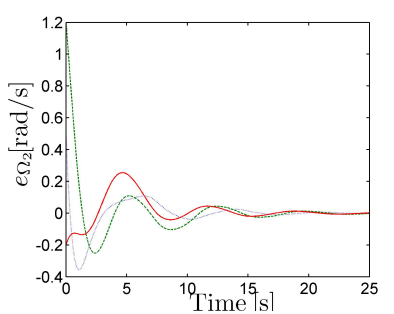

(b) Angular velocity tracking error $e_{\Omega_{1}}$
Fig. 4. Angular velocity tracking errors of the two spacecrafts. (First component: dotted, second component: dashed, third component: solid)

The control gains are chosen to be $k_{\Omega_{1}}=k_{\Omega_{2}}=3$, $k_{1}=0.7$.

Figures in (2) shows $\Psi_{1}$ and $\Psi_{2}$ achieving zero. The control inputs are shown in figures (3). Figure (4) shows position individual components of angular velocity errors $e_{\Omega_{1}}$ and $e_{\Omega_{2}}$ going to zero.

\section{CONCLUSION}

In this paper a new relative attitude trajectory tracking control law that achieves desired attitude trajectory, using LOS measurements is proposed. By combining a classical attitude determination scheme and more recent geometric control results, we have improved upon the result by $\mathrm{Wu}$ et al. (2013). The attitude control law obtained in terms of LOS vectors shows tracking of desired relative attitude trajectory even in presence of position dynamics. The desired equilibrium configuration is shown to be almost global asymptotic stable under the proposed control law.

\section{REFERENCES}

Andrle, M.S., Crassidis, J.L., Linares, R., Cheng, Y., and Hyun, B. (2009). Deterministic relative attitude determination of three-vehicle formations. Journal of guidance, control, and dynamics, 32(4), 1077-1088.

Bayadi, R. and Banavar, R.N. (2013). Almost global attitude stabilization of a rigid body for both internal and external actuation schemes. European Journal of Control.

Bernstein, D.S. (2009). Matrix mathematics: theory, facts, and formulas. Princeton University Press.

Bhat, S.P. and Bernstein, D.S. (2000). A topological obstruction to continuous global stabilization of rotational motion and the unwinding phenomenon. Systems \&6 Control Letters, 39(1), 63-70.

Bullo, F. and Murray, R.M. (1999). Tracking for fully actuated mechanical systems: A geometric framework. Automatica, 35(1), 17-34.

Chaturvedi, N.A. and McClamroch, N.H. (2006). Almost global attitude stabilization of an orbiting satellite including gravity gradient and control saturation effects. In American Control Conference, 2006, 6-pp. IEEE.

Khalil, H.K. (2002). Nonlinear systems, volume 3. Prentice hall Upper Saddle River.

Lee, T. (2012). Relative attitude control of two spacecraft on so (3) using line-of-sight observations. In American Control Conference (ACC), 2012, 167-172. IEEE. 
Mayhew, C.G. and Teel, A.R. (2011). Synergistic potential functions for hybrid control of rigid-body attitude. In American Control Conference (ACC), 2011, 875-880. IEEE.

Nair, S. and Leonard, N.E. (2007). Stable synchronization of rigid body networks. Networks and Heterogeneous Media, 2(4), 597.

Sanyal, A.K. and Chaturvedi, N.A. (2008). Almost global robust attitude tracking control of spacecraft in gravity. In Proceedings AIAA Guidance, Navigation and Control Conference.

Sarlette, A. (2009). Geometry and symmetries in coordination control.

Sarlette, A., Sepulchre, R., and Leonard, N. (2007). Cooperative attitude synchronization in satellite swarms: a consensus approach. In Proceedings of the 17th IFAC Symposium on Automatic Control in Aerospace.

Sarlette, A., Sepulchre, R., and Leonard, N.E. (2009). Autonomous rigid body attitude synchronization. Automatica, 45(2), 572-577.

Scharf, D.P., Hadaegh, F.Y., and Ploen, S.R. (2004). A survey of spacecraft formation flying guidance and control. part ii: control. In American Control Conference, 2004. Proceedings of the 2004, volume 4, 2976-2985. IEEE.

Warier, R. and Sinha, A. (2013). Los based attitude alignment of two spacecraft in formation. In 5th International Conference of Spacecraft Formation Flying Missions and Technologies, Munich.

Wertz, J.R. (1978). Spacecraft attitude determination and control, volume 73. Springer.

Wu, T.H., Flewelling, B., Leve, F., and Lee, T. (2013). Spacecraft relative attitude formation tracking on so (3) based on line-of-sight measurements. In American Control Conference (ACC), 2013, 4820-4825. IEEE. 